SCIENTIFIC REPORT

\title{
Ophthalmic artery blood flow in patients with internal carotid artery occlusion
}

\author{
T Yamamoto, K Mori, T Yasuhara, M Tei, N Yokoi, S Kinoshita, M Kamei
}

Br J Ophthalmol 2004;88:505-508. doi: 10.1136/bjo.2003.025809

Aim: To evaluate the risk factors for rubeosis iridis by colour Doppler imaging (CDI) in patients with complete internal carotid artery occlusion (ICAO).

Methods: 34 eyes of 32 consecutive patients with complete ICAO were enrolled. Using CDI, blood flow direction (forward, reverse, undetectable) in the ophthalmic artery (OA), central retinal artery (CRA), and short posterior ciliary artery (SPCA) were determined. Arterial mean blood velocity (Vmean) and resistive index (RI) were calculated and correlations between the rubeosis iridis incidence and CDI parameters analysed.

Results: The eyes were classified into four types according to blood flow direction: forward flow in OA, CRA, and SPCA (type 1; $n=11$ ); reverse OA and forward CRA and SPCA flow (type 2a; $n=12$ ); reverse $O A$ and undetectable CRA and SPCA flow (type 2b; $n=8$ ); undetectable flow in all three arteries (type $3 ; n=3$ ). Rubeosis iridis was seen only in type $2 b$ and 3 eyes. Type $2 b$ showed significantly $(p<0.01)$ higher Vmean and lower RI values in the OA, indicating more rapid reverse flow than in type $2 a$ eyes. Although in type 1 and $2 a$ eyes OA flow was in opposite directions, they manifested no rubeosis iridis and no difference in the Vmean and RI values of the CRA and SPCA.

Conclusions: The classification of eyes from patients with ICAO into four types by CDI may facilitate the identification of the eyes at high risk for rubeosis iridis. Markedly diminished flow in both the CRA and SPCA may result in rubeosis iridis, regardless of OA flow direction.

1 $\mathrm{n}$ patients with complete internal carotid artery occlusion (ICAO), chronic progressive ocular ischaemia ${ }^{1-5}$ leads to rubeosis iridis and neovascular glaucoma. To explain the underlying mechanisms, the following hypothesis has been proposed. When ICAO occurs in patients with incomplete blood circulation in the circle of Willis, the blood flow in the ophthalmic artery (OA) reverses to supply the ipsilateral brain. This so called "steal phenomenon" results in ocular ischaemia, ${ }^{6-8}$ whose prolongation leads to rubeosis iridis and neovascular glaucoma. It remains unclear, however, why some eyes deteriorate rapidly while others show no clinical symptoms of ocular ischaemia.

Colour Doppler imaging (CDI) is a non-invasive, repeatable technique for measuring orbital blood flow. ${ }^{10}$ The haemodynamic parameters of CDI are not sex dependent and do not vary between orbits. ${ }^{11}$ Since Lieb et al ${ }^{12}$ first reported CDI as a reliable means of evaluating ocular circulation, it has been used to measure retrobulbar blood flow in ICAO patients and to confirm the presence of the steal phenomenon. ${ }^{78}$ Mawn et $a l^{13}$ noted that the average peak systolic velocity in the CRA and SPCA was similar, regardless of OA flow direction, and that many patients with reverse OA flow demonstrated no evidence of ocular ischaemic syndrome.

To determine which eyes have a tendency to suffer from iris neovascularisation, we evaluated the ocular circulation by CDI in patients with complete ICAO with or without rubeosis iridis.

\section{PATIENTS AND METHODS}

Between April 1997 and December 1999, 34 eyes from 32 consecutive patients with ipsilateral complete ICAO were prospectively studied at Kyoto Prefectural University of Medicine. There were seven eyes from seven women and 27 eyes from 25 men; the patients averaged 66.5 years in age (range 51-79 years). All ICAO lesions diagnosed arteriographically by a neurologist were within $2 \mathrm{~cm}$ of the origin of the internal carotid artery. Of the 34 eyes, 14 were affected by right and 20 by left ICAO; in two patients both eyes were involved. Of the 32 patients, $20(62.5 \%)$ had hypertension, 13 $(40.6 \%)$ had diabetes, and seven $(21.9 \%)$ had coronary artery disease; 27 were referred from neurology or vascular surgery services, and five initially reported because of visual symptoms (amaurosis fugax, $\mathrm{n}=3$; visual loss, $\mathrm{n}=3$; and central scotoma, $\mathrm{n}=1$ ).

CDI was performed at the orbit ipsilateral to the ICAO with the patient in the supine position. We used a Toshiba SSA260A instrument (Toshiba Medical Co, Tokyo, Japan) and a 7.5 MHz transducer whose detection limits were $0.2 \mathrm{~mm}$ for the vessel diameter and $1 \mathrm{~cm} / \mathrm{s}$ for blood flow velocity. CDI was performed once at the initial examination at our department; the timing of the patients' ICAO was unknown. Informed consent was obtained from all patients.

Peak systolic velocity (Vs) and end diastolic velocity (Vd) were measured in the OA, the central retinal artery (CRA), and the short posterior ciliary arteries (SPCA). The OA was identified as the vessel parallel to the nasal border of the optic nerve just after crossing it; the CRA as the vessel within the optic nerve and approximately $2-5 \mathrm{~mm}$ behind the globe; and the SPCA as the vessel on the temporal side of the optic nerve approximately $10-15 \mathrm{~mm}$ behind the globe. The sample volume was $0.5 \mathrm{~mm}$ in width; the Doppler angle was adjusted to the vessels. In each artery, mean blood velocity (Vmean) and the resistive index (RI) values, indicative of downstream resistance, were calculated using the equation.

$$
\text { Vmean }=(\mathrm{Vs}+\mathrm{Vd}) / 2 \text { and } \mathrm{RI}=(\mathrm{Vs}-\mathrm{Vd}) / \mathrm{Vs}
$$

We performed three analyses to determine the risk factors for rubeosis iridis. Firstly, we analysed the relation between the rubeosis iridis incidence and the patients' clinical

\footnotetext{
Abbreviations: CDI, colour Doppler imaging; CRA, central retinal artery; ICAO, internal carotid artery occlusion; $O A$, ophthalmic artery; $\mathrm{RI}$, resistive index; SPCA, short posterior ciliary artery
} 
characteristics. We classified all 34 eyes into those with (group 1) and without rubeosis iridis (group 2) and compared the groups in terms of mean patient age ( $t$ test) and sex distribution, and incidence of severe contralateral internal carotid artery stenosis (ICAS) (>70\%) and hypertension, diabetes, and coronary artery disease (Fisher's exact probability test).

In the second analysis we established four eye types based on the retrobulbar blood flow direction (figs 1 and 2): forward OA, CRA, and SPCA flow (type 1); reverse OA and forward CRA and SPCA flow (type 2a); reverse OA and undetectable CRA and SPCA flow (type 2b); undetectable flow in all three arteries (type 3 ). We compared the four types in terms of the above mentioned clinical characteristics and investigated the incidence of rubeosis iridis among the four types.

In the third analysis, we compared average Vmean and RI values in the OA, CRA, and SPCA among the four eye types (Scheffé). Data were summarised by their mean (SD). p Values lower than 0.05 were considered significant.

\section{RESULTS}

Table 1 shows the clinical characteristics of patients with and without rubeosis iridis, and table 2 shows the clinical characteristics of each eye type. There was no statistically significant difference between the two groups and between eye types with respect to any of the clinical characteristics compared in this study.

Of the 34 eyes in this study, five (14.7\%) developed rubeosis iridis; four of eight type $2 \mathrm{~b}$ eyes and one of three type 3 eyes were affected.

Figure 3 compares the average Vmean and RI values of the OA flow in eyes of type $1,2 \mathrm{a}$, and $2 \mathrm{~b}$. No OA flow was detectable in type 3 eyes. The velocity of reverse OA flow (type $2 \mathrm{a}$ and $2 \mathrm{~b}$ ) is shown as negative values. The Vmean of the OA flow was significantly increased $(\mathrm{p}<0.01)$ in type 1 $(6.8(1.7) \mathrm{cm} / \mathrm{s}), 2 \mathrm{a}(20.3(7.0) \mathrm{cm} / \mathrm{s})$, and $2 \mathrm{~b}$ eyes $(36.1$ (5.6) $\mathrm{cm} / \mathrm{s})$, respectively. The RI of the OA flow was decreased in type $1(0.70(0.09)), 2 \mathrm{a}(0.59(0.13))$, and $2 \mathrm{~b}$ eyes $(0.18(0.27))$, respectively. The RI value of type $2 b$ eyes was significantly $(\mathrm{p}<0.01)$ lower than in type 1 and 2 a eyes and there was no significant difference $(p=0.35)$ between type 1 and 2 a eyes.

In type 1 and 2a eyes, the average Vmean and RI values in the CRA were as follows: type $1,4.9(2.3) \mathrm{cm} / \mathrm{s}$ and 0.65 (0.14), respectively; type $2 \mathrm{a}, 3.9(1.5) \mathrm{cm} / \mathrm{s}$ and $0.59(0.10)$, respectively. In the SPCA, these values were as follows: type $1,5.9(1.0) \mathrm{cm} / \mathrm{s}$ and $0.68(0.08)$, respectively; type $2 \mathrm{a}, 4.5$ $(2.5) \mathrm{cm} / \mathrm{s}$ and $0.70(0.16)$, respectively. Although in type 1 and $2 \mathrm{a}$ eyes OA flow was in opposite directions, they manifested no difference with respect to the Vmean and RI values of the CRA ( $p=0.21, p=0.20$, respectively) or SPCA ( $p=0.09, p=0.63$, respectively) (data not shown).

\section{DISCUSSION}

In patients with ICAO, we established four eye types according to the retrobulbar blood flow direction and compared them in terms of patient clinical characteristics and the incidence of rubeosis iridis. There was no statistically significant difference with respect to clinical characteristics among the four eye types. However, among 11 eyes classified as type $2 \mathrm{~b}$ and 3 , five $(45.5 \%)$ manifested rubeosis iridis; none of the 23 type 1 and 2a eyes were affected by rubeosis iridis.

We studied the blood flow in each eye type in detail. In type 1, the forward OA flow may have been preserved by collateral circulation via the circle of Willis in response to reduced inflow pressure in the distal internal carotid artery (ICA). ${ }^{78}$ The blood flow in the ocular end arteries was relatively well maintained, while the Vmean of the OA was
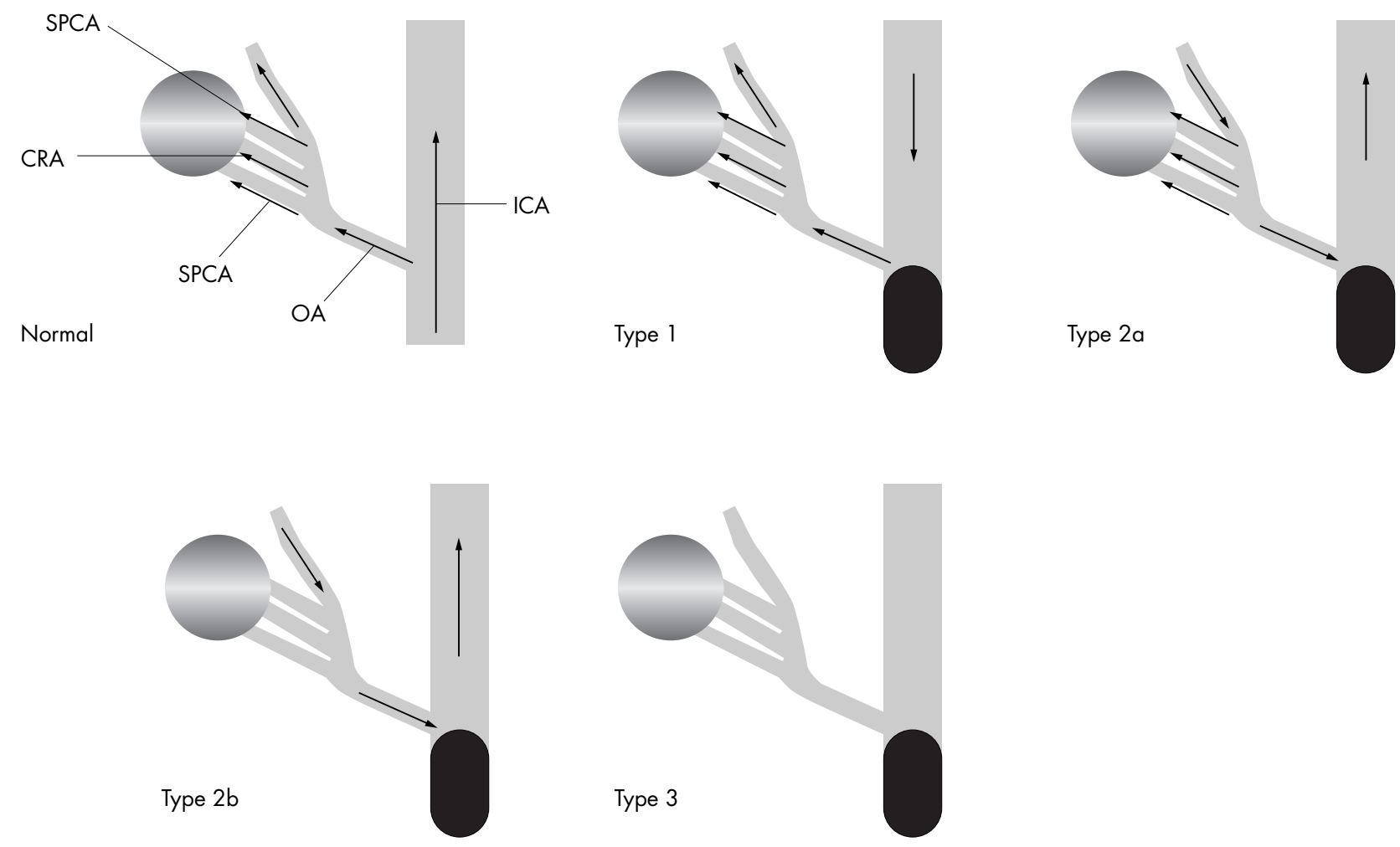

Figure 1 Four types of retrobulbar circulation. A modified classification previously reported by Costa et al. ${ }^{8}$ Normal = normal circulation in patients without ICAO; Type 1 = forward OA, CRA, and SPCA flow; Type $2 a=$ reverse OA and forward CRA and SPCA flow; Type $2 b=$ reverse OA and undetectable CRA and SPCA flow; Type 3 = undetectable OA, CRA, and SPCA flow. 

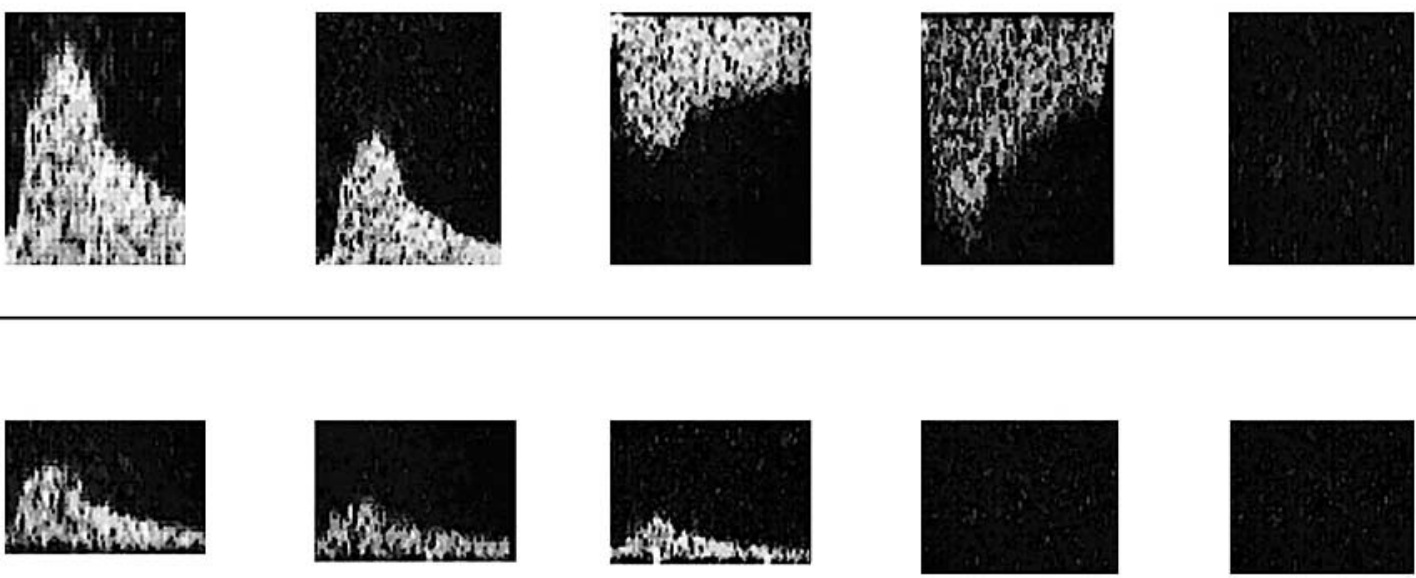

SPCA
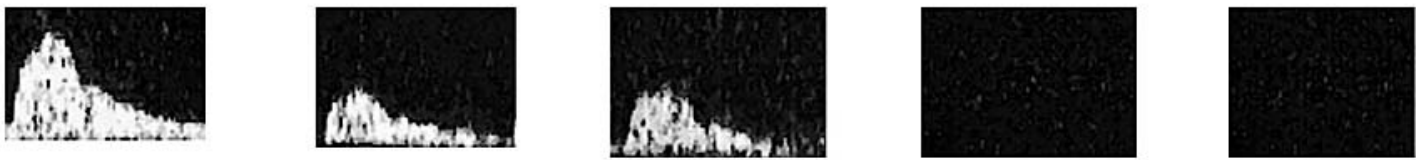

Figure $2 \mathrm{CDI}$ observations in the four types of circulation. Normal = normal circulation in patients without ICAO; Type $1=$ forward OA, CRA, and SPCA flow; Type $2 a=$ reverse OA and forward CRA and SPCA flow; Type $2 b=$ reverse OA and undetectable CRA and SPCA flow; Type $3=$ undetectable OA, CRA, and SPCA flow.

decreased. In type 2a eyes, the observed reverse OA flow represents collateralisation from the external carotid artery system in response to reduced inflow pressure in the OA. However, the blood flow in the ocular end arteries was relatively well maintained, suggesting that some of the reverse OA flow deviated towards the globe. In type $2 \mathrm{~b}$ eyes, almost the entire collateral circulation via the OA was deviated towards the internal carotid artery, reducing the blood flow to the eye. Rubeosis iridis occurred in four eyes of type $2 \mathrm{~b}(50 \%)$. In type 3 , no collateral blood supply developed. Therefore, the ocular end circulation was markedly reduced, possibly because of insufficient collateral circulation via both the circle of Willis and the external carotid artery system. Rubeosis iridis was seen in one eye of type $3(33 \%)$.

Our results indicate that of 34 eyes, $23(67.6 \%)$ maintained a relatively good blood supply via collateral circulation; all of these were classified as type 1 and 2 a eyes. Eleven of the 34

Table 1 Clinical characteristics of patients with or without rubeosis iridis

\begin{tabular}{|c|c|c|c|}
\hline & $\begin{array}{l}\text { Rubeosis iridis } \\
(+) \\
(n=5)\end{array}$ & $\begin{array}{l}\text { Rubeosis iridis } \\
(-1) \\
(n=29)\end{array}$ & p Value \\
\hline Age (years) & $70.2(7.2)$ & $66.5(9.2)$ & $0.397^{*}$ \\
\hline Sex & 5 men & $\begin{array}{l}7 \text { women, } \\
22 \text { men }\end{array}$ & $0.290+$ \\
\hline $\begin{array}{l}\text { Contralateral ICAS } \\
(>70 \%)\end{array}$ & 3 & 8 & 0.179 \\
\hline Hypertension & 4 & 17 & 0.353 \\
\hline Diabetes & 3 & 11 & 0.328 \\
\hline $\begin{array}{l}\text { Coronary artery } \\
\text { disease }\end{array}$ & 3 & 5 & 0.072 \\
\hline
\end{tabular}

ICAS $=$ internal carotid artery stenosis; ${ }^{*}$ test; †Fisher's exact probability test. eyes were classified as type $2 \mathrm{~b}$ and 3 ; all manifested insufficient blood supply and $45.5 \%$ of these eyes developed rubeosis iridis. We found that reverse OA flow does not produce rubeosis iridis in all ICAO patients, and that markedly diminished CRA and SPCA flow may result in rubeosis iridis regardless of the OA flow direction. Our observation supports the hypothesis of Mawn et $a l^{13}$ that eyes are highly adaptive to proximal blood flow reduction.

In our comparison of CDI parameters, the Vmean of the OA flow was increased in eye types $1,2 a$, and $2 b$, in ascending order, while the RI value was decreased. This suggests that forward OA flow (type 1) results in low Vmean and high RI values, indicating high resistance flow towards the OA branches. Reverse OA flow (types $2 \mathrm{a}$ and $2 \mathrm{~b}$ ), on the other hand, results in high Vmean and low RI values, representing low resistance flow towards the internal carotid artery. ${ }^{14}$

Type $2 \mathrm{~b}$ eyes showed significantly higher Vmean and lower RI values in the OA than did type 2 a eyes. This suggests that the reverse OA flow in type 2a supplies blood not only to the distal ICA (low resistance), but also to the ocular end arteries (high resistance), while the reverse OA flow in type $2 \mathrm{~b}$ eyes mainly supplies blood to the distal ICA. Therefore, eyes with rapid reverse OA flow may be at greater risk for ocular ischaemic syndrome.

The Vmean and RI values of the CRA and SPCA showed no statistically significant difference between type 1 and 2a eyes in which the OA flow was in the opposite direction. This suggests that in patients with forward CRA and SPCA flow and reverse OA flow (type 2a) almost the same blood supply is maintained as in eyes with forward OA flow (type 1). Reverse OA flow in type 2a eyes represents the major supplier of ocular end circulation and compensates for the reduced blood flow.

Patients with ICAO usually are subjected to anastomosis of the superficial temporal and middle cerebral arteries (STAMCA bypass). The primary aim of this procedure is 

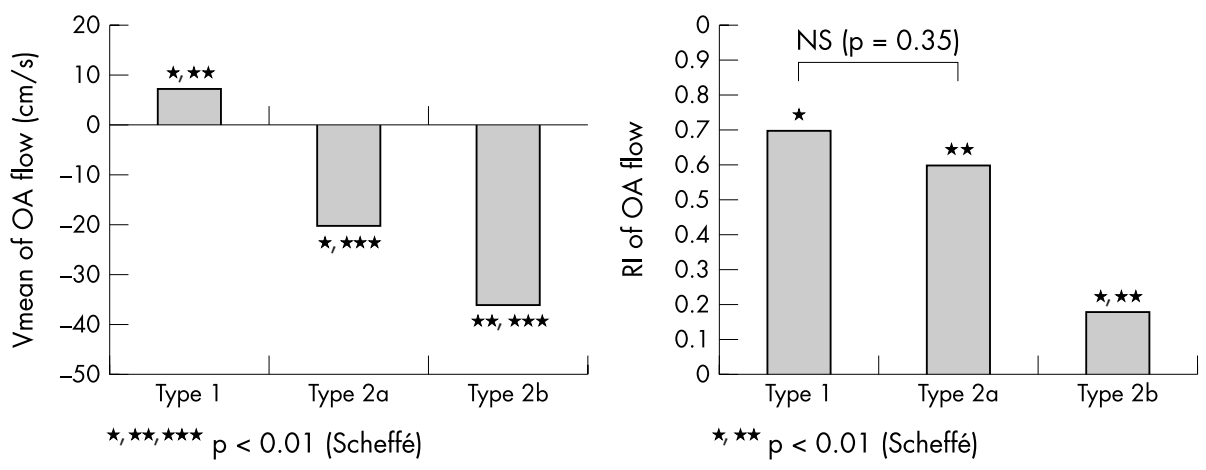

Figure $3 \mathrm{CDI}$ parameters in ICAO patients with ophthalmic artery flow (mean). Vmean, mean blood velocity; $\mathrm{RI}=$ resistive index; $\mathrm{OA}=$ ophthalmic artery; NS = not significant.

Table 2 Clinical characteristics of four patient types

\begin{tabular}{|c|c|c|c|c|c|}
\hline & Type $1(n=11)$ & Type 2a $(n=12)$ & Type $2 b(n=8)$ & Type $3(n=3)$ & p Value \\
\hline Age (years) & $64.2(10.7)$ & $66.9(9.3)$ & $70.9(5.8)$ & $67.7(5.7)$ & $0.468^{*}$ \\
\hline Sex & 3 women, 8 men & 3 women, 9 men & 1 woman, 7 men & 3 men & $0.673+$ \\
\hline $\begin{array}{l}\text { Contralateral ICAS } \\
(>70 \%)\end{array}$ & 2 & 4 & 4 & 1 & 0.541 \\
\hline Hypertension & 6 & 8 & 5 & 2 & 0.941 \\
\hline Diabetes & 2 & 4 & 4 & 2 & 0.323 \\
\hline Coronary artery disease & 2 & 2 & 3 & 0 & 0.507 \\
\hline
\end{tabular}

improvement of ipsilateral brain ischaemia by providing for collateral blood supply from the external carotid artery. This treatment reportedly led to improved ocular ischaemia. ${ }^{15-17}$ Shibuya et $a l^{16}$ posited that collateral blood flow to the hemisphere through the bypass reduces theft from the ophthalmic circulation, resulting in improved blood flow to the eye. However, there is currently no consensus on the beneficial effects of this surgery on the eye in ICAO patients.

Reverse OA flow does not invariably result in blood theft from the eye. Some patients with reverse OA flow (our type 2a) do not experience rubeosis iridis. Patients with markedly diminished CRA and SPCA flow (that is, no development of collateral blood supply to the ocular end arteries) eventually suffer rubeosis iridis. Therefore, they should be treated with STA-MCA bypass surgery to avoid the development of neovascular glaucoma.

Over the course of a 2 year follow up period, none of our ICAO patients developed new rubeosis iridis. While our observation period is too short and the study population too small to draw firm conclusions, we posit that undetectable flow is not indicative of an absence of flow because CDI assesses flow velocity rather than blood flow volume. Despite these reservations, our study suggests that measurement and classification of the orbital circulation by CDI facilitates the identification of eyes at high risk for rubeosis iridis, and provides important information on indications for STAMCA bypass surgery in patients with ipsilateral complete ICAO.

\section{ACKNOWLEDGEMENTS}

The authors thank Ms Ursula Petralia for her valuable contribution to the editing of this manuscript.

\section{Authors' affiliations}

T Yamamoto, K Mori, T Yasuhara, M Tei, N Yokoi, S Kinoshita, Department of Ophthalmology, Kyoto Prefectural University of Medicine, Kyoto, Japan

M Kamei, Department of Ophthalmology, Osaka University Medical School, Osaka, Japan
Correspondence to: Takami Yamamoto, MD, Department of Ophthalmology, Kyoto Prefectural University of Medicine, 465 Kajiicho, Hirokoii-agaru, Kawaramachi-dori, Kamigyo-ku, Kyoto 602-0841, Japan; tyamamot@ophth.kpu-m.ac.jp

Accepted for publication 1 August 2003

\section{REFERENCES}

1 Brown GC, Magargal LE. The ocular ischemic syndrome. Int Ophthalmol $1988 ; 11: 239-51$

2 Huckman MS, Hass S. Reversed flow through the ophthalmic artery as a cause of rubeosis iridis. Am J Ophthalmol 1972;74:1094-9.

3 Carter JB. Chronic ocular ischemia and carotid vascular disease. Stroke $1985 ; 16: 721-8$

4 Sturrock GD, Mueller HR. Chronic ocular ischaemia. Br J Ophthalmol 1984:68:716-23.

5 Sivalingam A, Brown GC, Magargal LE. The ocular ischemic syndrome. Visual prognosis and the effect of treatment. Int Ophthalmol 1991;15:15-20.

6 Reynolds PS, Greenberg JP, Lien LM, et al. Ophthalmic artery flow direction on color flow duplex imaging is highly specific for severe carotid stenosis. J Neuroimaging 2002;12:5-8

7 Costa VP, Kuzniec S, Molnar U, et al. Clinical findings and hemodynamic changes associated with severe occlusive carotid artery disease. Ophthalmology 1997; 104:1994-2002.

8 Costa VP, Kuzniec S, Molnar U, et al. Collateral blood supply through the ophthalmic artery. A steal phenomenon analyzed by color Doppler imaging. Ophthalmology 1998;105:689-93.

9 Quaranta L, Harris A, Donato F, et al. Color Doppler imaging of ophthalmic artery blood flow velocity. A study of repeatability and agreement. Ophthalmology 1997; 104:653-8.

10 Lieb WE, Cohen SM, Merton DA, et al. Color Doppler imaging of the eye and orbit. Technique and normal vascular anatomy. Arch Ophthalmol $1991 ; 109: 527-31$

11 Greenfield DS, Heggerick PA, Hedges TR. Color Doppler imaging of normal orbital vasculature. Ophthalmology 1995;102:1598-605.

12 Lieb WE, Flaharty PM, Sergott RC, et al. Color Doppler imaging provides accurate assessment of orbital blood flow in occlusive carotid artery disease. Ophthalmology 1991;98:548-52.

13 Mawn LA, Hedges TR, Rand W, et al. Orbital color Doppler imaging in carotid occlusive disease. Arch Ophthalmol 1997:115:492-6.

14 Ho AC, Lieb WE, Flaharty PM, et al. Color Doppler imaging of the ocular ischemic syndrome. Ophthalmology 1992;99:1453-62.

15 Katz B, Weinstein PR. Improvement of photostress recovery testing after extracranial-intracranial bypass surgery. Br J Ophthalmol 1986;70:277-80.

16 Shibuya M, Suzuki Y, Takayasu M, et al. Effects of STA-MCA anastomosis for ischaemic oculopathy due to occlusion of the internal carotid artery. Acta Neurochir 1990;103:71-5.

17 Guthikonda M, Guyot LL, Diaz FG. Future of extracranial-intracranial bypass. Neurol Res 2002;24(Suppl 1):S80-3. 